\title{
REVIEW
}

\section{Current and Future Therapies for Idiopathic Pulmonary Fibrosis}

Paolo Spagnolo $\cdot$ Francesco Bonella $\cdot$ Martina Vasakova $\cdot$ Michael Kreuter $\cdot$

Toby M. Maher

To view enhanced content go to www.pulmonarytherapy-open.com

Received: October 23, 2015 / Published online: December 10, 2015

(c) The Author(s) 2015. This article is published with open access at Springerlink.com

\section{ABSTRACT}

Idiopathic pulmonary fibrosis (IPF) is the most common form of fibrosing idiopathic interstitial pneumonia. The disease, which primarily occurs in older adults, is inexorably progressive with a 5-year survival of

Electronic supplementary material The online version of this article (doi:10.1007/s41030-015-0009-4) contains supplementary material, which is available to authorized users.

P. Spagnolo $(\varangle)$

Medical University Clinic, Canton Hospital

Baselland, Liestal, Switzerland

e-mail: paolo.spagnolo@ksbl.ch

P. Spagnolo

University of Basel, Liestal, Switzerland

F. Bonella

Interstitial and Rare Lung Disease Unit,

Ruhrlandklinik, University Hospital, University of

Duisburg-Essen, Essen, Germany

M. Vasakova

Department of Respiratory Medicine of the 1st

Medical School, Thomayer Hospital, Videnska,

Prague, Czech Republic

\section{Kreuter}

Center for Interstitial and Rare Lung Diseases, Pneumology and Respiratory Critical Care Medicine, Thoraxklinik, University of Heidelberg, Heidelberg, Germany approximately 20\%. Improved understanding of disease pathobiology has affected the approach to treatment. Indeed, originally thought to be a chronic inflammatory disorder, IPF is now considered the result of persistent alveolar epithelial micro-injury followed by an aberrant repair response. This paradigm shift along with significant improvement in disease definition and patient stratification has led to an exponential increase in the number of high-quality clinical trials,

\section{Kreuter}

Translational Lung Research Center Heidelberg (TLRCH), Member of the German Center for Lung Research (DZL), Heidelberg, Germany

\section{T. M. Maher}

National Institute for Health Research Biological Research Unit, Royal Brompton Hospital, London, UK

\section{T. M. Maher}

National Heart and Lung Institute, Imperial College, London, UK 
most of which, however, have produced negative results, probably due to the multitude of cell types, growth factors and signaling pathways involved in the fibrotic process. Therefore, until recently IPF has lacked effective therapies. Finally, in 2014, pirfenidone, a compound with broad antifibrotic, anti-inflammatory and antioxidant properties and nintedanib, an orally available, small-molecule tyrosine kinase inhibitor with selectivity for vascular endothelial growth factor, platelet-derived growth factor and fibroblast growth factor receptors, have shown to significantly slow functional decline and IPF disease progression with an acceptable safety profile. This is a major step forward. However, neither pirfenidone nor nintedanib is a cure for IPF; neither drug improves lung function and the disease continues to progress in most patients despite treatment. A number of agents with high potential are currently being tested and many more are ready for clinical trials. Their completion is critical for achieving the ultimate goal of curing this devastating disease.

Keywords: Guidelines; Idiopathic pulmonary fibrosis; Nintedanib; Pirfenidone; Therapy; Treatment; Usual interstitial pneumonia

\section{DISEASE OVERVIEW}

Idiopathic pulmonary fibrosis (IPF) is the most common form of chronic, progressive, fibrosing interstitial pneumonia [1]. The disease, which occurs primarily in older adults, is limited to the lungs and is associated with the radiological and/or histopathologic pattern of usual interstitial pneumonia (UIP) [2, 3]. Radiological UIP is characterized on high-resolution computed tomography (HRCT) by the presence of reticular opacities, often associated with traction bronchiectasis, typically in a basal, subpleural and patchy distribution [4]. However, a definite diagnosis of UIP on HRCT requires the presence of honeycombing, which manifests as clustered cystic airspaces, usually of comparable diameter on the order of 3-10 mm (Fig. 1) [5]. Histopathologic UIP consists of a combination of fibrotic areas with scarring and honeycomb change alternate with areas of less affected or even normal lung parenchyma (Fig. 2). The fibrotic zones are composed mainly of dense collagen, although scattered subepithelial foci of proliferating fibroblasts and myofibroblasts (so-called fibroblastic foci) are a consistent finding. Notably, a pattern of UIP can be found in a number of conditions including, among others, connective tissue disease (CTD), chronic hypersensitivity pneumonitis (HP) and pneumoconioses (especially asbestosis), which makes it essential a rigorous and multidisciplinary approach to the diagnosis of IPF (e.g., idiopathic UIP) [6]. This point remains unappreciated by many physicians, and, as a

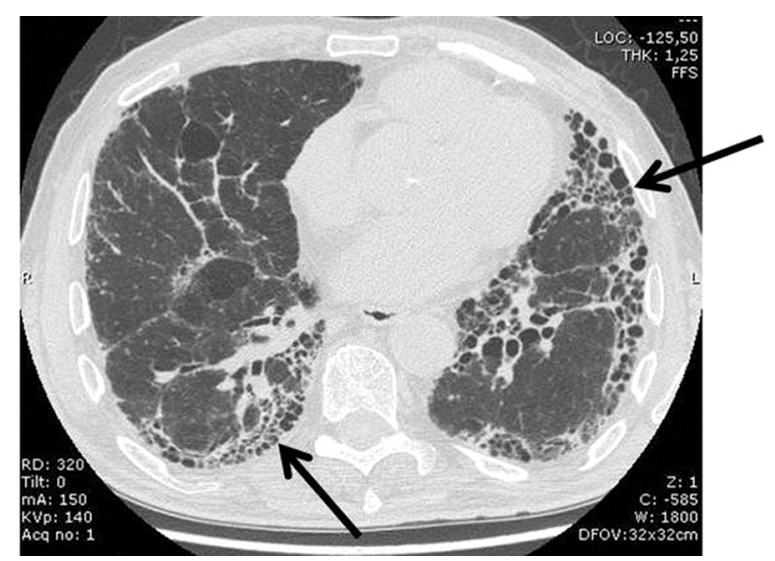

Fig. 1 Usual interstitial pneumonia pattern. Chest high-resolution computed tomography showing reticular abnormality in a typical subpleural distribution. Areas with honeycombing (arrow) are also seen 


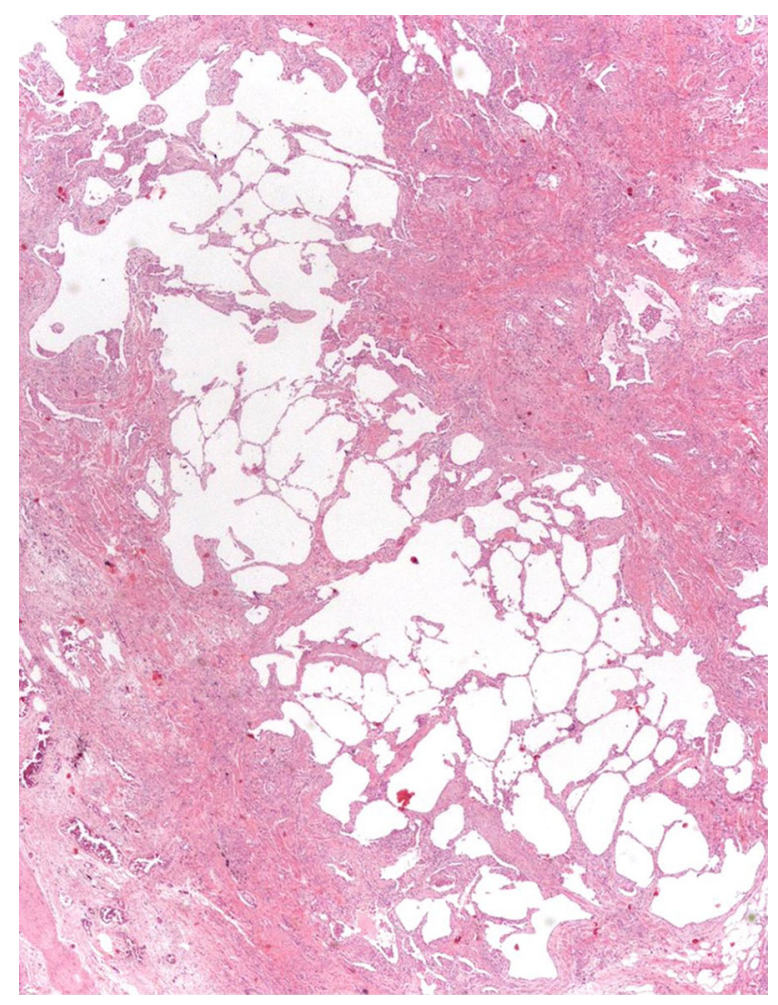

Fig. 2 Surgical lung biopsy specimen showing usual interstitial pneumonia, characterized by the abrupt transition from dense fibrosis (top right and bottom left) to nearly normal lung (center). Hematoxylin-eosin, $20 \times$. Courtesy of Giulio Rossi, MD, Modena, Italy

result, patients are often incompletely evaluated and empirically treated as IPF.

Incidence and prevalence of IPF increase dramatically with age. Indeed, the disease is virtually absent in patients younger than 50 years of age, but is present in an estimated $0.2 \%$ of those older than 75 years of age $[7,8]$. The underlying causes of the fibrotic response in IPF remain unknown. However, cigarette smoking, exposure to metal and wood dust, microbial agents, chronic microaspiration secondary to gastro-oesophageal reflux and genetic abnormalities have all been associated with an increased risk of developing the disease [9-13]. Consistent with the progressive nature of the fibrotic process, shortness of breath on exertion is the most common presenting symptom. IPF is almost invariably fatal with a 5 -year survival of approximately $20 \%$ and a mortality burden higher than that of many cancers [14]. Yet, its clinical course and rate of progression are highly variable and unpredictable. In fact, periods of relative stability may be punctuated by episodes of accelerated deterioration, so-called 'acute exacerbations' often resulting in respiratory failure and death $[15,16]$. Moreover, a subgroup of patients, predominantly smoking males, display an accelerated clinical course and have a gene expression pattern that is different from those with slower progression and longer survival [17].

Our understanding of IPF has undergone dramatic change in the last two decades, and this has affected the approach to treatment. Indeed, older pathogenetic models of chronic active inflammation leading to fibrosis have evolved to current models of repeated alveolar epithelial cell micro-injury and dysregulated repair response in the presence of relatively little inflammation [18]. According to the abnormal repair response hypothesis, key pathogenetic events include uncontrolled proliferation of lung fibroblasts and differentiation of fibroblasts into myofibroblasts, which excessively deposit extracellular matrix (ECM) proteins in the interstitial space leading to progressive scarring of the lung, architectural distortion and irreversible loss of function [19-21]. As a result, more recent clinical trials of pharmacological interventions have evaluated the efficacy of molecules targeting the wound healing cascade and fibrogenesis, although, overall, with disappointing results, probably because of the multitude of mediators, growth factors and signaling pathways involved in the fibrotic process [22]. Finally, more recently, two compounds with pleiotropic mechanisms of 
action-pirfenidone and nintedanib-have consistently proven effective in reducing functional decline and disease progression in patients with IPF $[23,24]$. In this article we summarize the evidence supporting the efficacy of pirfenidone and nintedanib in IPF and provide an overview of clinical drug development in this devastating disease. This article is based on previously conducted studies and does not involve any new studies of human or animal subjects performed by any of the authors.

\section{GUIDELINES ON TREATMENT OF IPF}

The management of patients with IPF is largely based on the recommendations of the guidelines developed by the American Thoracic Society (ATS)/European Respiratory Society (ERS)/Japanese Respiratory Society (JRS)/Latin American Thoracic Association (ALAT) [2]. This evidence-based document, which was published in 2011, has recently been updated to incorporate reappraisal of previously evaluated treatment options and recommendations on novel agents [25]. For each outcome of interest, a multidisciplinary panel assessed the overall certainty (e.g., the confidence) in effect estimate according to the GRADE (Grading of Recommendations Assessment, Development and Evaluation) methodology [26], which is based on the following criteria: risk of bias, precision, consistency, directness of the evidence, risk for publication bias, presence of dose-effect relationship, magnitude of effect, and assessment of the effect of plausible residual confounding or bias. The confidence in effect estimates for each outcome was graded as high, moderate, low, or very low [25]. In addition, for any given question, the multidisciplinary committee made a recommendation for or against [27]. The recommendations were either 'strong' or 'conditional', based on the following factors: quality and strength of evidence, outcomes and associated importance to patients, desirable and undesirable consequences of treatment, costs, implications of treatment on health equity, feasibility of treatment, the acceptability of treatment to stakeholders and implementation issues. Current recommendations are summarized in Table 1.

Three treatment interventions, which are discussed in the following sections, received a conditional recommendation for use (e.g., pirfenidone, nintedanib, and antiacid medication), although the committee emphasized that recommendations with the same rating should not by default be considered equivalent.

\section{PIRFENIDONE}

Pirfenidone (5-methyl-1-phenylpyridin-2[1H]one) is an orally available, synthetic, non-peptide, low molecular weight molecule with anti-fibrotic, anti-inflammatory and anti-oxidant properties [28]. Pirfenidone's biological actions include inhibition of the synthesis and activity of transforming growth factor (TGF)- $\beta$; inhibition of fibroblast proliferation and collagen secretion; reduction of pro-fibrotic gene expression in response to fibrotic stimuli; and inhibition of pro-inflammatory cytokine (mainly tumor necrosis factor $[\mathrm{TNF}]-\alpha)$ production and release [29-32].

Following promising results from an open-label trial [33], safety and efficacy of pirfenidone in patients with IPF were initially 
Table 1 Overview of recommendations in the 2015 and 2011 IPF guidelines

\begin{tabular}{|c|c|c|}
\hline Agent/intervention & 2015 guidelines & 2011 guidelines \\
\hline Nintedanib & Conditional recommendation for use $\mathrm{a}^{\mathrm{a}}$ & Not addressed \\
\hline Pirfenidone & Conditional recommendation for use $\mathrm{a}^{\mathrm{a}}$ & Conditional recommendation against use $\mathrm{e}^{\mathrm{b}}$ \\
\hline Antiacid therapy & Conditional recommendation for use ${ }^{c}$ & Conditional recommendation for use $\mathrm{a}^{\mathrm{a}}$ \\
\hline Bosentan, macitentan & Conditional recommendation against use $\mathrm{b}^{\mathrm{b}}$ & Strong recommendation against use ${ }^{\mathrm{a}}$ \\
\hline NAC monotherapy & Conditional recommendation against $u e^{b}$ & Conditional recommendation against use $\mathrm{e}^{\mathrm{b}}$ \\
\hline Sildenafil & Conditional recommendation against use $\mathrm{a}^{\mathrm{a}}$ & Not addressed \\
\hline Ambrisentan & Strong recommendation against use $^{\mathrm{b}}$ & Not addressed \\
\hline $\begin{array}{l}\text { Combination prednisone, } \\
\text { azathioprine and NAC }\end{array}$ & Strong recommendation against use ${ }^{b}$ & Conditional recommendation against use $\mathrm{e}^{\mathrm{b}}$ \\
\hline Imatinib & Strong recommendation against use $^{\mathrm{a}}$ & Not addressed \\
\hline Warfarin & Strong recommendation against use $^{\mathrm{a}}$ & Conditional recommendation against use $\mathrm{e}^{\mathrm{c}}$ \\
\hline Therapy for IPF-associated PH & $\begin{array}{l}\text { Reassessment of the previous } \\
\text { recommendation was deferred }\end{array}$ & Conditional recommendation against use $\mathrm{e}^{\mathrm{c}}$ \\
\hline $\begin{array}{l}\text { Lung transplantation: single } \\
\text { versus bilateral }\end{array}$ & $\begin{array}{l}\text { Formulation of a recommendation } \\
\text { was deferred }\end{array}$ & Not addressed \\
\hline
\end{tabular}

Adapted from [25]

$I P F$ Idiopathic pulmonary fibrosis, $N A C \mathrm{~N}$-acetylcysteine, $P H$ Pulmonary hypertension

a Moderate confidence in effect estimates

b Low confidence in effect estimates

c Very low confidence in effect estimates

evaluated in a phase 2 double-blind, placebo-controlled, multicenter study conducted in Japan by Azuma et al. [34]. This trial was stopped prematurely following an interim analysis revealing that episodes of acute exacerbation of IPF (AE-IPF) had occurred exclusively in the placebo arm $(n=5$; $14 \%$ vs. $0 \%$ in the pirfenidone arm; $P=0.0031$ ) during the 9-month study period. The change in the lowest oxygen saturation (SpO2) during a 6-min exercise test (6MET), the primary endpoint, did not reach statistical significance $(P=0.0722)$. However, in a pre-specified analysis of patients who maintained a SpO2 $>80 \%$ during a $6 \mathrm{MET}$ at baseline, the lowest $\mathrm{SpO} 2$ during a 6MET improved in the pirfenidone group at 6 and 9 months
( $P=0.0069$ and $P=0.0305$, respectively). Positive treatment effects were also demonstrated in the change in vital capacity $(\mathrm{VC})$ at 9 months $(P=0.0366)$. Following these promising data, Taniguchi and colleagues conducted a multicenter, double-blind, placebo-controlled phase three study in which 275 Japanese patients were randomized in a 2:1:2 ratio to high-dose $(1800 \mathrm{mg} /$ day $)$ or low-dose $\quad(1200 \mathrm{mg} /$ day $)$ pirfenidone, or placebo [35]. The study met its primary endpoint of change in $\mathrm{VC}$ from baseline to week 52. In fact, the rate of decline of VC was lower in both the high-dose $(-0.09 \mathrm{~L})$ and low-dose pirfenidone arms $(-0.08 \mathrm{~L})$ compared to the placebo arm $(-0.16 \mathrm{~L} ; P=0.042$ and $P=0.039$, respectively). Significant differences 
were also observed in progression-free survival time (defined as time to death and/or $\geq 10 \%$ decline in VC from baseline) between the high-dose and the placebo arms $(P=0.028)$ and in change in total lung capacity (TLC) between the low-dose and the placebo arms $(P=0.040)$. However, a limitation of this study was that the primary endpoint was changed before unblinding, which possibly hampered the integrity of the study. Overall, pirfenidone was well tolerated. The most common drug-related adverse event was photosensitivity (observed in 51\% of patients in the high-dose group and 53\% in the low-dose group), which was mild in severity in most cases and not a major reason for discontinuation of the study. Pirfenidone was approved in Japan for use in patients with IPF in 2008.

The CAPACITY (Clinical Studies Assessing Pirfenidone in IPF: Research on Efficacy and Safety Outcomes; ClinicalTrials.gov identifiers, NCT00287729 and NCT00287716) program consisted of two almost identical randomized, double-blind, placebo-controlled, multinational phase 3 trials (PIPF-004 and PIPF-006) that involved 110 centers across Europe, North America, Mexico, and Australia [36]. Both trials enrolled patients aged 40-80 years with a diagnosis of IPF made within the previous 48 months based on clinical, radio-logic and/or pathologic data, and according to the 2000 ATS/ ERS guidelines [1]. Inclusion criteria included also predicted forced VC (FVC) of at least 50\%, predicted diffusing capacity of the lung for carbon monoxide $\left(\mathrm{DL}_{\mathrm{CO}}\right)$ of at least $35 \%$, either predicted FVC or predicted $\mathrm{DL}_{\mathrm{CO}}$ of $90 \%$ or less, and 6-min walk test distance (6MWD) of at least $150 \mathrm{~m}$. In study 004, 435 patients were assigned in a 2:1:2 dosing ratio to pirfenidone $2403 \mathrm{mg} /$ day $\quad(n=174), \quad$ pirfenidone $1197 \mathrm{mg} /$ day $(n=87)$, or placebo $(n=174)$, whereas study 006 had only two arms (i.e., pirfenidone $2403 \mathrm{mg} /$ day, $n=173$ and placebo, $n=171$ ). Pirfenidone was administered daily with food in three divided doses and titrated up to the full dose over 2 weeks. The primary endpoint of both studies was change in percentage predicted FVC from baseline to week 72 and efficacy analyses, which were performed in the intent-to-treat population, used a rank analysis of covariance (ANCOVA) model. In PIPF-004, pirfenidone reduced decline in FVC. In fact, mean FVC change at week 72 was $-8.0 \%$ in the pirfenidone $2403 \mathrm{mg}$ /day group and $-12.4 \%$ in the placebo group $(P=0.001)$, whereas in the pirfenidone $1197 \mathrm{mg} /$ day arm, the primary outcome was intermediate to that of the pirfenidone $2403 \mathrm{mg} /$ day and placebo arms. In addition, 35 of 174 (20\%) patients in the pirfenidone arm and 60 of 174 (35\%) in the placebo arm had an FVC decline of at least $10 \%(P=0.001)$. By contrast, in study 006, the between-group difference in FVC change at week 72 was not significant $(P=0.501)$, although, a consistent pirfenidone effect was apparent until week 48 $(P=0.005)$ and in an analysis of all study time points $(P=0.007)$. Mean change in percentage predicted FVC was $-9.0 \%$ in the pirfenidone arm and $-9.6 \%$ in the placebo arm. Most common pirfenidone-related adverse events included nausea (36\% vs. $17 \%$ in placebo), dyspepsia (19\% vs. $7 \%)$, vomiting (14\% vs. $4 \%)$, anorexia ( $11 \%$ vs. $4 \%)$, photosensitivity ( $12 \%$ vs. $2 \%)$, skin rash (32\% vs. $12 \%)$, and dizziness (18\% vs. 10\%). However, they were generally mild to moderate in severity, reversible, and without clinically significant sequelae. These trials had sufficient methodological quality to be included in a Cochrane meta-analysis that showed that pirfenidone significantly reduces the rate of functional decline and risk of disease progression compared with placebo [37]. 
In 2011, pirfenidone has been approved for the treatment of IPF in Europe, but not by the US Food and Drug Administration (FDA), which requested an additional phase 3 study to confirm efficacy. In the ASCEND (Assessment of Pirfenidone to Confirm Efficacy and Safety in IPF; ClinicalTrials.gov identifier, NCT01366209) study, 555 patients with IPF were randomly assigned to receive either pirfenidone $2403 \mathrm{mg} /$ day $(n=278)$ or placebo $(n=277)$ for 52 weeks [23]. Of note, to enroll patients at higher risk for disease progression, certain aspects of the CAPACITY study design were modified such as exclusion of patients with major airflow limitation (ratio of the forced expiratory volume in one second (FEV1) to $\mathrm{FVC}<0.80$ ) and reduction of the minimum baseline $\mathrm{DL}_{\mathrm{CO}}$ from $35 \%$ to $30 \%$ of the predicted value. With regard to the HRCT diagnostic criteria for inclusion, only a definite UIP pattern was accepted. The study met its primary outcome of change from baseline to week 52 in the percentage of predicted FVC. In addition, a significant relative reduction of $47.9 \%$ in the proportion of patients who had an absolute decline of $\geq 10 \%$ in percentage predicted FVC or who died [46 patients (16.5\%) vs. 88 patients (31.8\%); $P<0.001]$, and a significant relative increase of $132.5 \%$ in the proportion of patients with no decline in FVC [63 patients (22.7\%) vs. 27 patients (9.7\%); $P<0.001]$ was seen in the pirfenidone compared to the placebo arm. A series of sensitivity analyses confirmed the robustness of these findings and the estimated magnitude of pirfenidone effect (e.g., an approximate 50\% reduction in FVC decline) in patients with IPF [38]. Pirfenidone treatment reduced also the decline in the 6MWD $(P=0.04)$ and improved progression-free survival (e.g., time to the first occurrence of any one of the following: a confirmed decrease of $\geq 10 \%$ in the percentage of the predicted FVC, a confirmed decrease of $50 \mathrm{~m}$ or more in the $6 \mathrm{MWD}$, or death; $P<0.001)$. No significant differences between pirfenidone and placebo were found in dyspnea scores $(P=0.16)$ or in all-cause $(4.0 \%$ vs. $7.2 \%$; $P=0.10)$ or IPF-related mortality $(1.1 \%$ vs. $2.5 \% ; \quad P=0.23)$. However, a pre-specified pooled analysis from the ASCEND and CAPACITY trials showed that pirfenidone significantly reduced both death from any cause [3.5\% vs. $6.7 \%$; hazard ratio (HR) 052; $P=0.01]$ and from IPF (1.1\% vs. $3.5 \%$; HR 0.32; $P=0.006)$ compared with placebo at week 52 . Similar to the CAPACITY trials, gastrointestinal and skin-related events were more common in the pirfenidone group than in the placebo group, although they were generally mild to moderate in severity, reversible, and without clinically significant sequelae. Moreover, the proportion of adverse events leading to discontinuation of study treatment did not differ between the pirfenidone (40 patients; $14.4 \%$ ) and the placebo groups (30 patients; $10.8 \%)$.

Recommendations on optimal management of pirfenidone-related adverse events based on existing guidelines, research evidence, and expert opinion have recently been published [39]. In October 2014 the FDA granted pirfenidone fast track, priority review, orphan product, and breakthrough designation. Several reports have confirmed long-term favorable safety and efficacy profiles of pirfenidone [40-42]. In particular, an interim analysis of RECAP, an ongoing open-label, long-term, follow-up extension study that included patients who completed the CAPACITY or ASCEND trials, showed that almost $50 \%$ of the patients initially randomized to pirfenidone were still receiving therapy after 5-year follow-up [41]. 


\section{NINTEDANIB}

Nintedanib is a potent inhibitor of the receptor tyrosine kinases vascular endothelial growth factor receptor (VEGFR) 1-3, fibroblast growth factor receptor (FGFR) 1-3, and platelet-derived growth factor receptor (PDGFR) a and b [43]. By competitively and reversibly inhibiting the adenosine triphosphate binding pocket of the receptor tyrosine kinases VEGFR, FGFR, and PDGFR, nintedanib blocks the intracellular signaling needed for the proliferation, migration and transformation of fibroblasts [44, 45]. However, it has been recently shown that antifibrotic properties of nintedanib also include: inhibition of TGF- $\beta$ receptor(s) signaling; inhibition of fibronectin and collagen 1a1 mRNA expression independent of TGF- $\beta$ signaling; and induction of non-canonical autophagy [46].

The safety and efficacy of oral nintedanib in patients with IPF were initially evaluated in the TOMORROW (To Improve Pulmonary Fibrosis With BIBF 1120; ClinicalTrials.gov identifier, NCT00514683), a phase 2, dose-finding, 12-month, randomized, double-blind, placebo-controlled trial [47]. Inclusion criteria included age $\geq 40$ years, IPF diagnosed within the previous 5 years, FVC of $\geq 50 \%$ of predicted value, $\mathrm{DL}_{\mathrm{CO}}$ of $30-79 \%$ of predicted value, and HRCT of the chest performed within the previous 12 months. In the TOMORROW trial, four different doses of nintedanib were tested [i.e., $50 \mathrm{mg}$ once a day $(n=86)$, and $50 \mathrm{mg}$ $(n=86), 100 \mathrm{mg}(n=86)$, or $150 \mathrm{mg}(n=85)$ all twice a day] against placebo $(n=85)$. Nintedanib showed a trend towards a reduction in the annual rate of decline in FVC, the primary endpoint. Specifically, the adjusted annual rate of decline in FVC was $0.06 \mathrm{~L} /$ year in the group receiving nintedanib $150 \mathrm{mg}$ twice daily and $0.19 \mathrm{~L} /$ year in the placebo group corresponding to a reduction of $68.4 \%$ in the rate of FVC loss $[P=0.06$ using a closed testing procedure for multiplicity correction (primary analysis) and $P=0.01$ using hierarchical testing, both pre-specified] [47]. In addition, compared with placebo, significantly fewer patients in the nintedanib $150 \mathrm{mg}$ twice daily group had a decline in mean FVC of $\geq 10 \%$ or $\geq 200 \mathrm{~mL} \quad(23.8 \% \quad$ vs. $44.0 \%$, respectively; $P=0.004)$. The highest dose of nintedanib was also associated with a lower incidence of AE-IPF (2.4 vs. 15.7 per 100-patient-years; risk ratio: $0.16 ; P=0.02$ ) and an improved quality of life as assessed by St. George's Respiratory Questionnaire (SGRQ; -0.66 vs. +5.46 points; $P=0.007)$ compared with placebo. Overall, nintedanib showed an acceptable safety profile. The most frequent adverse event in the group receiving $150 \mathrm{mg}$ nintedanib twice daily was diarrhea $(55.3 \%$ vs. $15.3 \%$ in the placebo group), followed by nausea $(23.5 \%$ vs. $9.4 \%)$ and vomiting (12.9\% vs. $4.7 \%)$. The adverse events most frequently leading to discontinuation were also diarrhea, nausea, and vomiting, but the proportion of patients who discontinued the study medication because of adverse events did not differ between the nintedanib $150 \mathrm{mg}$ twice daily group and the placebo group. Clinically significant elevations in liver enzyme levels (e.g., at least three times the upper limit of the normal range for aspartate aminotransferase or alanine aminotransferase at any time after baseline) were observed in 6 patients in the group receiving $150 \mathrm{mg}$ of nintedanib twice a day $(7.1 \%)$ and none in the placebo group; however, only two patients discontinued the study medication because of persistently abnormal liver function tests.

The INPULSIS (INPULSIS-1 and INPULSIS-2; ClinicalTrials.gov identifiers, NCT01335464 and NCT01335477, respectively) trials were 
two parallel 52-week, randomized, double-blind, placebo-controlled, phase three studies designed to confirm the efficacy and safety of nintedanib $150 \mathrm{mg}$ twice daily in patients with IPF [24]. The eligibility criteria for patients with IPF were identical to those of the TOMORROW trial. A total of 1066 patients were randomized 3:2 to receive nintedanib $150 \mathrm{mg}$ twice daily $(n=309$ in INPULSIS- 1 and $n=329$ in INPULSIS-2) or placebo $(n=204$ in INPULSIS- 1 and $n=219$ in INPULSIS-2). In both trials, nintedanib significantly reduced the rate of decline in FVC over the 52-week study period (the primary end point). Indeed, the adjusted annual rate of change in FVC was $-114.7 \mathrm{~mL}$ in the nintedanib arm and $-239.9 \mathrm{~mL}$ in the placebo arm in INPULSIS-1 (between-group difference: $125.3 \mathrm{~mL} ; \quad P<0.001)$ and -113.6 and $-207.3 \mathrm{~mL}$ in INPULSIS-2 (between-group difference: $93.7 \mathrm{~mL} ; \quad P<0.001)$, respectively. Pre-specified sensitivity analyses confirmed the robustness of the results of the primary analysis. Moreover, in both trials, patients in the nintedanib arm were more likely than those in the placebo arm to be stable at week 52 (e.g., to have a decline in percentage predicted FVC of no greater than $5 \% ; 52.8 \%$ vs. $38.2 \%$ in INPULSIS- $1, P=0.001$; and $53.2 \%$ vs. $39.3 \%$ in INPULSIS-2, $P=0.001)$. Overall, the risk of progression (e.g., absolute decline in percent predicted FVC of $\geq 10 \%$ or death) was significantly reduced with nintedanib versus placebo by $47 \%$ in INPULSIS-1 $(24.3 \%$ vs. $40.7 \%$; HR $0.53 ; \quad P=0.0001), \quad 33 \% \quad$ in INPULSIS-2 $\quad(29.8 \%$ vs. $42.0 \%$; HR 0.67 ; $P=0.0054)$ and $40 \%$ in the pooled analysis (27.1\% vs. 41.4\%; HR 0.60; $P<0.0001$ ) [45]. As for the two key secondary end points (i.e., the time to the first AE-IPF as reported by a site investigator and the change from baseline in the total score on the SGRQ), the two trials provided mixed results. In fact, the time to the first AE-IPF was significantly increased in INPULSIS-2 (HR 0.38, $P=0.005$ ), but not in INPULSIS-1 (HR 1.15, $P=0.67$ ). However, a pre-specified sensitivity analysis of pooled data from INPULSIS-1 and INPULSIS-2 showed that the time to first adjudicated AE-IPF (confirmed or suspected) was significantly increased with nintedanib compared to placebo (HR 0.32, $P=0.001$ ). In INPULSIS-2, at week 52 there was a significantly smaller increase in the total SGRQ score (consistent with less deterioration in health-related quality of life) in the nintedanib group than in the placebo group (2.80 points in the nintedanib group vs. 5.48 points in the placebo group; $P=0.02$ ), whereas in INPULSIS-1 there was no significant between-group difference in the adjusted mean change in the SGRQ total score from baseline to week 52 (4.34 points vs. 4.39 points; $P=0.97)$. Similarly, in a pre-specified pooled analysis of the two trials, the adjusted mean change from baseline in the SGRQ total score did not significantly differ between the nintedanib and placebo arms. In a pre-specified pooled analysis, there was no significant between-group difference in death from any cause $(5.5 \%$ vs. $7.8 \%$; HR 0.70 ; $P=0.14)$ or death from a respiratory cause (3.8\% vs. $5.0 \%$; HR: $0.74 ; P=0.34$ ). Of note, subgroups analyses of pooled data from the INPULSIS trials showed that sex, age $(<65$, $\geq 65$ years), race (White, Asian), baseline FVC $\%$ predicted $(\leq 70 \%,>70 \%)$, baseline SGRQ total score $(\leq 40,>40)$, smoking status (never, ex/ current), systemic corticosteroid use (yes, no), and bronchodilator use (yes, no) did not influence the efficacy of nintedanib on the decline in FVC over 52 weeks [48]. Similar to the TOMORROW trial, the most frequent adverse event in the nintedanib groups in both INPULSIS- 1 and INPULSIS- 2 was diarrhea (approximately 60\% within the first 3 months 
of treatment), which overall led to premature discontinuation in $4.4 \%$ of patients in the nintedanib group [49]. However, in both trials, the proportion of patients with serious adverse events was similar in the nintedanib and placebo groups.

Similar to pirfenidone, in October 2014 nintedanib has been granted fast track, priority review, orphan product, and breakthrough designation by the FDA, and has been licensed in Europe in early 2015.

\section{ANTIACID THERAPY}

Gastroesophageal reflux (GER), including clinically silent GER, is highly prevalent in patients with IPF [50, 51], and markers of aspiration (e.g., bile acids and pepsin) are significantly elevated in bronchoalveolar lavage fluid (BALF) from patients with IPF compared to patients with interstitial lung disease other than IPF and healthy controls [52]. In addition, levels of inflammatory biomarkers such as lactate dehydrogenase, alkaline phosphatase, C-reactive protein, and TNF- $\alpha$ are higher in BALF of patients with newly diagnosed IPF compared to patients with newly diagnosed GER disease [53]. Chronic microaspiration secondary to GER is considered a risk factor for the development or worsening of the disease, suggesting that prevention of microaspiration could have an important role in the management of IPF.

In an uncontrolled retrospective study of 204 patients with IPF, antiacid treatment (AAT) was associated with reduced radiological fibrosis and was an independent predictor of longer survival time [54]. In addition, a post hoc analysis of data from patients assigned to placebo arms in three IPFnet-sponsored randomized-controlled trials (RCTs) revealed that patients taking AAT at baseline [either proton-pump inhibitors or $\mathrm{H}_{2}$ blockers; $n=124 / 242$ (51\%)] had a smaller decrease in FVC at 30 weeks compared with those not taking AAT $(P=0.05)$ after adjusting for sex, baseline percentage predicted FVC, and baseline percentage predicted $\mathrm{DL}_{\mathrm{CO}}$ [55]). However, a more recent post hoc analysis of patients in the placebo arms of three large RCTs $[n=624,291$ of whom $(47 \%)$ were taking AAT at baseline] showed that AAT did not improve progression-free survival (defined as FVC decrease $\geq 10 \%$, $6 \mathrm{MWD}$ decrease $\geq 50 \mathrm{~m}$, or death), FVC decline, hospitalization and all-cause and IPF-related mortality, but was associated with a significantly higher rate of all-cause hospitalization (HR 1.4, $P=0.042$ ) [56]. Despite the lack of evidence from prospective RCTs, the 2015 guidelines recommend AAT for most patients with IPF based on the potential benefit and the favorable side effect profile of antiacid medications [25]. However, further research focusing on efficacy and long-term safety of AAT in patients with IPF is clearly needed.

\section{MOST DEVELOPED COMPOUNDS FOR IPF}

Historically, the pharmaceutical industry has been reluctant to invest in research and development of drugs for rare diseases like IPF [57]. The last decade has witnessed a growing interest in IPF and a willingness among stakeholders to work together to develop and fund clinical trials of novel compounds. In addition, improved understanding of disease pathogenesis has resulted in an exponentially increasing number of potential therapeutic targets. Selected most developed compounds for IPF are listed below. 


\section{PRM-151}

PRM-151 (Promedior, Inc, Lexington, MA, USA) is a recombinant form of an endogenous human protein, pentraxin-2 (PTX-2), that is specifically active at the site of tissue damage. PRM-151 is an agonist that binds to Fc-gamma receptors on monocytes and induces their differentiation into regulatory (pro-resolutive) macrophages, thus promoting epithelial healing and resolution of inflammation and scarring. In a multiple ascending dose, phase 1 study in patients with IPF, PRM-151 was safe and well tolerated, and demonstrated a trend towards improvement in pulmonary function and circulating levels of surfactant protein D and VEGF at 8 weeks [58]. PRM-151 is currently being tested in IPF in a phase 2 randomized, placebo-controlled trial (ClinicalTrials.gov identifier, NCT02550873).

\section{IW001}

Ongoing adaptive immune response against autoantigens is thought to play an important role in disease progression in some IPF patients [59]. Type $\mathrm{V}$ collagen $(\operatorname{col}(\mathrm{V}))$ is a minor collagen normally sequestered within the lung interstitium and, therefore, hidden from the immune system. Lung injury may lead to col(V) exposure, making it available for activation of an autoimmune response [60]. A sustained autoimmune response against $\operatorname{col}(\mathrm{V})$, which is present in approximately $50 \%$ of patients, may result in abnormal lung remodeling and fibrotic changes [61, 62]. In a recently published phase 1 study (ClinicalTrials.gov identifier, NCT01199887), an orally available compound that induces immune tolerance to $\operatorname{col}(\mathrm{V})$ (IW001; ImmuneWorks, Inc, Indianapolis, IN, USA), was safe and well tolerated, and showed a trend towards stabilization of FVC and metalloproteinase 7 levels in anti-col(V) $\mathrm{Ab}^{+}$ patients with IPF [63]. Further study with $\operatorname{col}(\mathrm{V})$ oral immunotherapy is warranted to confirm the therapeutic effect of IW001 in patients with IPF.

\section{TD139}

Galectin-3, a member of the galectin family of galactoside binding lectins, has been shown to play a central role in fibrosis development and progression through the activation of macrophages and recruitment and activation of myofibroblasts [64]. TD139 (Galecto Biotech $\mathrm{AB}$, Copenhagen, Denmark) is a highly potent, specific inhibitor of the galactoside binding pocket of galectin-3 formulated for inhalation. Safety and tolerability of TD139 in patients with IPF are currently being evaluated in a phase $1 b /$ 2a trial (ClinicalTrials.gov identifier, NCT02257177).

\section{BMS-986020}

Lysophosphatidic acid (LPA) is a well-recognized mediator of wound healing and tissue fibrosis [65], and LPA receptor 1 (LPA1) appears to contribute to the development of IPF by inducing epithelial cell apoptosis, fibroblast recruitment and vascular leak [66]. The safety and efficacy of BMS-986020 (Bristol-Myers Squibb, New York, NY, USA), a high-affinity, selective, small-molecule antagonist of LPA1 in patients with IPF is currently being assessed in a phase 2 trial (ClinicalTrials.gov identifier, NCT01766817).

\section{FG-301}

It is a humanized monoclonal antibody directed against connective tissue growth factor (CTGF), 
a key mediator of tissue remodeling and fibrosis. Safety and tolerability of FG-3019 (FibroGen, Inc, San Francisco, CA, USA) in patients with IPF have been evaluated in an exploratory phase 2 dose escalation open-label study. Data of the first dose cohort $(15 \mathrm{mg} / \mathrm{kg}$ intravenous every 3 weeks) have been published in the form of an abstract [67]. Fifty-three subjects were enrolled and treated; 39 of them completed the treatment period and 14 withdrew. FG-3019 was safe and well tolerated. In addition, the majority of patients [27 of the 38 (71\%) who had acceptable data at week 48] experienced improvement or $<5 \%$ loss in FVC \% predicted at week 48. Moreover, improved or stable lung fibrosis as measured by quantitative HRCT was observed in more than half of subjects. On average, patients with improved or stable fibrosis also had improved pulmonary function. Based on these encouraging data, a randomized placebo-controlled phase 2 trial in patients with IPF is currently being conducted (ClinicalTrials.gov identifier, NCT01890265).

\section{Lebrikizumab}

Lebrikizumab (Hoffmann-La Roche, Basel, Switzerland) is a humanized monoclonal antibody against interleukin (IL)-13, a Th2 cytokine that is found at increased levels in the lungs of patients with IPF [68]. In vitro, IL-13 stimulates fibroblast proliferation and induces CCL6/C10, a chemotactic factor for mononuclear phagocytes, which in turn are an important source of growth factors that regulate ECM synthesis [69]. In vivo, IL-13 overexpression results in increased fibrosis in mice in response to bleomycin [69]. Lebrikizumab (as both monotherapy and as combination therapy with pirfenidone background therapy) is currently being tested in patients with IPF in a phase $2 b$ study
(ClinicalTrials.gov identifier, NCT01872689) with results expected in late 2016 .

\section{Tralokinumab}

Tralokinumab (MedImmune LLC, Gaithersburg, MD, USA) is a human IL-13-neutralizing immunoglobulin G4 monoclonal antibody. Murray and colleagues have recently shown that the IL-13 pathway is significantly enhanced in biopsy samples from IPF patients who exhibit a rapidly progressing disease course compared with patients with a slower rate of lung function loss. In addition, they also demonstrated that inhibition of human IL-13 with tralokinumab attenuated established lung fibrosis and promoted alveolar epithelial repair processes in a humanized severe combined immunodeficiency (SCID) mouse model of IPF [70]. Safety, tolerability and effectiveness of multiple doses of tralokinumab are being evaluated in phase 2 studies (ClinicalTrials.gov identifiers, NCT01629667 and NCT02036580, the latter only in Japanese patients), which are ongoing yet currently not recruiting patients.

\section{SAR156597}

IL-4, a cytokine structurally related to IL-13, has also been implicated in the abnormal proliferation of fibroblasts that characterizes IPF [71]. In addition, targeting the IL-13 and IL-4 receptors has been shown to modulate the abnormal proliferative properties of human lung fibroblasts [72]. SAR156597 (Sanofi S.A., Paris, France) is a humanized bispecific antibody that neutralizes both IL-4 and IL-13 circulating cytokines. In patients with IPF, SAR156597 has been shown to be generally safe and well tolerated [73] The safety and efficacy of two dose levels of SAR156597 administered subcutaneously during 52 weeks 
in patients with IPF are currently being tested in a phase 2 study (ClinicalTrials.gov identifier, NCT02345070).

\section{Simtuzumab}

Simtuzumab (Gilead Sciences, Foster city, CA, USA) is a humanized monoclonal antibody against lysyl oxidase-like 2 (LOXL2), an enzyme that catalyzes covalent cross linking of ECM molecules, including collagen fibers. LOXL2 protein expression is observed in the fibroblastic foci and collagenous regions of diseased IPF lung tissue [74] and serum LOXL2 levels are associated with increased risk for IPF disease progression [75]. Moreover, in the bleomycin-induced mouse model of pulmonary fibrosis, inhibition of LOXL2 resulted in a marked reduction in activated fibroblasts, decreased production of growth factors and cytokines and decreased TGF- $\beta$ pathway signaling [74]. The safety and efficacy of simtuzumab in patients with IPF are being evaluated in a phase 2 study (ClinicalTrials.gov identifier, NCT01769196), which is ongoing, but currently not recruiting patients.

\section{STX-100}

STX-100 (Biogen, Weston, MA, USA) is a humanized monoclonal antibody against the integrin $\alpha \mathrm{v} \beta 6$, which functions as a key mediator of TGF- $\beta$ activation and plays an important functional role in promoting and maintaining fibrogenesis and epithelial injury [76]. In murine bleomycin-induced pulmonary fibrosis, partial inhibition of $\alpha v \beta 6$ effectively inhibits TGF- $\beta$ activation, epithelial injury, and tissue fibrosis [77]. The safety and tolerability of subcutaneously administered multiple, escalating doses of STX-100 in patients with IPF are being evaluated in a phase 2 study, which is currently recruiting participants (ClinicalTrials.gov identifier, NCT01371305).

\section{CONCLUSIONS}

IPF is a progressive and almost invariably fatal disease. Over the last decade, our knowledge of the mechanisms involved in disease pathobiology has substantially improved and this had allowed a number of clinical trials of pharmacological interventions to be undertaken and completed. This massive effort of the medical and industry community has produced the approval of two drugs of comparable safety and efficacy profile, pirfenidone and nintedanib, which will soon become standard of care worldwide (Table 2).

Yet, this is only the beginning as neither pirfenidone nor nintedanib is a cure for IPF, neither drug improves lung function and most patients continue to progress while on treatment. These limitations notwithstanding, the concerted effort by the scientific, professional and patient community as well as the pharmaceutical industry has the potential to finally develop a real cure for patients suffering from this devastating disease.

\section{ACKNOWLEDGMENTS}

No funding or sponsorship was received for this study or publication of this article. All named authors meet the International Committee of Medical Journal Editors (ICMJE) criteria for authorship for this manuscript, take responsibility for the integrity of the work as a whole, and have given final approval to the version to be published.

Disclosures. Dr Spagnolo serves as consultant for F. Hoffmann-La Roche Ltd and 
Table 2 Pirfenidone versus nintedanib: justification and implementation considerations according to the 2015 Idiopathic Pulmonary Fibrosis guidelines

Pirfenidone
High value on the potential benefit on patient-reported
outcomes such as disease progression as measured by rate
of FVC decline and mortality
Lower value on potentially significant adverse events and
cost of treatment
Pooled analysis of the CAPACITY and ASCEND trials
suggested improved mortality (relative risk: 0.70; $95 \%$ CI:
$0.47-1.02$; moderate confidence)
Pirfenidone treatment was associated with increased rates of
photosensitivity (high confidence), fatigue (moderate
confidence), stomach discomfort (moderate confidence),
and anorexia (high confidence). Patients should be
informed and educated on all potential adverse effects
Pirfenidone is a very costly intervention and this should be
considered in the decision-making process

It is unknown whether the therapeutic benefits would differ in patients with a more severe lung function impairment $(\mathrm{FVC}<50 \%)$ or in those with other comorbidities

Optimal duration of treatment and treatment effect duration while on therapy are unknown

\section{Nintedanib}

High value on the potential benefit on patient-reported outcomes such as disease progression as measured by rate of FVC decline and mortality

Lower value on potentially significant adverse events and cost of treatment

Pooled analysis of the TOMORROW and INPULSIS trials suggested improved mortality (relative risk: $0.70 ; 95 \% \mathrm{CI}$ : 0.47-1.03; moderate confidence)

Significantly more patients treated with nintedanib reported adverse events (high confidence), but not serious adverse events (high confidence). Patients should be informed and educated on all potential adverse effects

Costs of nintedanib should be considered in the decision-making process and this may limit its feasibility and use

It is unknown whether the therapeutic benefits would differ in patients with a more severe lung function impairment $(\mathrm{FVC}<50 \%)$ or in those with other comorbidities

Optimal duration of treatment and treatment effect duration while on therapy are unknown

Adapted from [25]

$C I$ Confidence interval, FVC Forced vital capacity

Santhera and has received consulting fees from InterMune International AG, Boehringer Ingelheim and Novartis. Dr Bonella has received grants and honoraria from $F$. Hoffmann-La Roche Ltd, InterMune International AG, Boehringer Ingelheim, Serendex and Sekisui Diagnostics. Dr Vasakova has received research grants and speaker and advisory board fees from InterMune International AG and F. Hoffmann-La Roche Ltd. Dr Kreuter and his institution have received grants from InterMune International AG and Boehringer Ingelheim. Dr Kreuter has received personal fees from InterMune International $A G$ and Boehringer Ingelheim. Dr Maher is in receipt of unrestricted academic industry grants from GSK, UCB and Novartis. Dr Maher or his institution have received advisory board or consultancy fees from Boehringer Ingelheim, Bayer, Biogen, ProMedior, Galapagos, GSK, InterMune International AG, Novartis, Lanthio, F. Hoffmann-La Roche Ltd, Takeda, Sanofi-Aventis and UCB. Dr Maher has received speaker's fees from UCB, Boehringer Ingelheim, InterMune International $A G$ and AstraZeneca. 
Compliance with Ethics Guidelines. This article is based on previously conducted studies, and does not involve any new studies of human or animal subjects performed by any of the authors.

Open Access. This article is distributed under the terms of the Creative Commons Attribution-NonCommercial 4.0 International License (http://creativecommons.org/licenses/ by-nc/4.0/), which permits any noncommercial use, distribution, and reproduction in any medium, provided you give appropriate credit to the original author(s) and the source, provide a link to the Creative Commons license, and indicate if changes were made.

\section{REFERENCES}

1. American Thoracic Society. Idiopathic pulmonary fibrosis: diagnosis and treatment. International consensus statement. American thoracic society (ATS), and the European respiratory society (ERS). Am J Respir Crit Care Med. 2000;161:646-64.

2. Raghu G, Collard HR, Egan JJ, et al. Idiopathic pulmonary fibrosis: evidence based guidelines for diagnosis and management. Am J Respir Crit Care Med. 2011;183:788-824.

3. Spagnolo P, Sverzellati N, Rossi G, et al. Idiopathic pulmonary fibrosis: an update. Ann Med. 2015;47:15-27.

4. Johkoh T, Muller NL, Cartier Y, et al. Idiopathic interstitial pneumonias: diagnostic accuracy of thin-section CT in 129 patients. Radiology. 1999;211:555-60.

5. Hansell DM, Bankier AA, MacMahon H, McLoud TC, Müller NL, Remy J. Fleischner society: glossary of terms for thoracic imaging. Radiology. 2008;246:697-722.

6. Wuyts WA, Cavazza A, Rossi G, Bonella F, Sverzellati N, Spagnolo P. Differential diagnosis of usual interstitial pneumonia: when is it truly idiopathic? Eur Respir Rev 2014; 23:308-319. Erratum in: Eur Respir Rev 2014; 23:537.
7. Raghu G, Weycker D, Edelsberg J, Bradford WZ, Oster G. Incidence and prevalence of idiopathic pulmonary fibrosis. Am J Respir Crit Care Med. 2006;174:810-6.

8. Raghu G, Chen S-Y, Yeh W-S, et al. Idiopathic pulmonary fibrosis in US Medicare beneficiaries aged 65 years and older: incidence, prevalence and survival, 2001-2011. Lancet Respir Med. 2014;2:566-72.

9. Iwai K, Mori T, Yamada N, Yamaguchi M, Hosoda Y. Idiopathic pulmonary fibrosis: epidemiologic approaches to occupational exposure. Am J Respir Crit Care Med. 1994;150:670-5.

10. Olson AL, Swigris JJ. Idiopathic pulmonary fibrosis: diagnosis and epidemiology. Clin Chest Med. 2012;33:41-50.

11. Spagnolo P, Grunewald J, du Bois RM. Genetic determinants of pulmonary fibrosis: evolving concepts. Lancet Respir Med. 2014;2:416-28.

12. Bellaye PS, Kolb M. Why do patients get idiopathic pulmonary fibrosis? Current concepts in the pathogenesis of pulmonary fibrosis. BMC Med. 2015;13:176.

13. Behr J, Kreuter M, Hoeper MH, et al. Management of patients with idiopathic pulmonary fibrosis in clinical practice: INSIGHTS-IPF registry. Eur Respir J. 2015;46:186-96.

14. Bjoraker JA, Ryu JH, Edwin MK, et al. Prognostic significance of histopathologic subsets in idiopathic pulmonary fibrosis. Am J Respir Crit Care Med. 1998;157:199-203.

15. Ley B, Collard HR, King TE Jr. Clinical course and prediction of survival in idiopathic pulmonary fibrosis. Am J Respir Crit Care Med. 2011;183:431-40.

16. Johannson KA, Ley B, Collard HR. Models of disease behavior in idiopathic pulmonary fibrosis. BMC Med. 2015;13:165.

17. Selman M, Carrillo G, Estrada A, et al. Accelerated variant of idiopathic pulmonary fibrosis: clinical behavior and gene expression pattern. PLoS ONE. 2007;2:e482.

18. $\mathrm{du}$ Bois RM. Strategies for treating idiopathic pulmonary fibrosis. Nat Rev Discov. 2010;9:129-40.

19. Selman M, King TE. Pardo A; American thoracic society; European Respiratory society; American college of chest physicians. Idiopathic pulmonary fibrosis: prevailing and evolving hypotheses about its pathogenesis and implications for therapy. Ann Intern Med. 2001;134:136-51. 
20. Wolters PJ, Collard HR, Jones KD. Pathogenesis of idiopathic pulmonary fibrosis. Annu Rev Pathol. 2014;9:157-79.

21. Spagnolo P, Rossi G, Cavazza A. Pathogenesis of idiopathic pulmonary fibrosis and its clinical implications. Expert Rev Clin Immunol. 2014;10:1005-17.

22. Maher TM. Idiopathic pulmonary fibrosis: pathobiology and novel approaches to treatment. Clin Chest Med. 2012;33:69-83.

23. King TE Jr, Bradford WZ, Castro-Bernardini S, et al. A phase 3 trial of pirfenidone in patients with idiopathic pulmonary fibrosis. $\mathrm{N}$ Engl J Med. 2014;370:2083-92.

24. Richeldi L, du Bois RM, Raghu G, et al. Efficacy and safety of nintedanib in idiopathic pulmonary fibrosis. N Engl J Med. 2014;370:2071-82.

25. Raghu G, Rochwerg B, Zhang Y, et al. An official ATS/ERS/JRS/ALAT clinical practice guideline: treatment of idiopathic pulmonary fibrosis. An update of the 2011 clinical practice guideline. Am J Respir Crit Care Med. 2015;192:e3-19.

26. Balshem $H$, Helfand $M$, Schünemann $H J$, et al. GRADE guidelines: 3. Rating the quality of evidence. J Clin Epidemiol. 2011;64:401-6.

27. Andrews JC, Schünemann $\mathrm{HJ}$, Oxman $\mathrm{AD}$, et al. GRADE guidelines: 15. Going from evidence to recommendation-determinants of a recommendation's direction and strength. J Clin Epidemiol. 2013;66:726-35.

28. Kim ES, Keating GM. Pirfenidone: a review of its use in idiopathic pulmonary fibrosis. Drugs. 2015;75:219-30.

29. Di Sario A, Bendia E, Svegliati Baroni G, et al. Effect of pirfenidone on rat hepatic stellate cell proliferation and collagen production. J Hepatol. 2002;37:584-91.

30. Schaefer CJ, Ruhrmund DW, Pan L, Seiwert SD, Kossen K. Antifibrotic activities of pirfenidone in animal models. Eur Respir Rev. 2011;20:85-97.

31. Nakayama S, Mukae $H$, Sakamoto $N$, et al. Pirfenidone inhibits the expression of HSP47 in TGF-beta1-stimulated human lung fibroblasts. Life Sci. 2008;82:210-7.

32. Oku H, Nakazato H, Horikawa T, Tsuruta Y, Suzuki R. Pirfenidone suppresses tumor necrosis factor-alpha, enhances interleukin-10 and protects mice from endotoxic shock. Eur J Pharmacol. 2002;446:167-76.
33. Raghu G, Johnson WC, Lockhart D, Mageto Y. Treatment of idiopathic pulmonary fibrosis with a new antifibrotic agent, pirfenidone: results of a prospective, open-label Phase II study. Am J Respir Crit Care Med. 1999;159:1061-9.

34. Azuma A, Nukiwa T, Tsuboi E, et al. Double-blind, placebo-controlled trial of pirfenidone in patients with idiopathic pulmonary fibrosis. Am J Respir Crit Care Med. 2005;171:1040-7.

35. Taniguchi $\mathrm{H}$, Ebina $\mathrm{M}$, Kondoh $\mathrm{Y}$, et al. Pirfenidone in idiopathic pulmonary fibrosis. Eur Respir J. 2010;35:821-9.

36. Noble PW, Albera C, Bradford WZ, et al. Pirfenidone in patients with idiopathic pulmonary fibrosis (CAPACITY): two randomised trials. Lancet. 2011;77:1760-9.

37. Spagnolo P, Del Giovane C, Luppi F. Non-steroid agents for idiopathic pulmonary fibrosis. Cochrane Database Syst Rev. 2010;9:CD003134.

38. Lederer DJ, Bradford WZ, Fagan EA, et al. Sensitivity analyses of the change in FVC in a phase 3 trial of pirfenidone for idiopathic pulmonary fibrosis. Chest. 2015;148:196-201.

39. Costabel U, Bendstrup E, Cottin V, et al. Pirfenidone in idiopathic pulmonary fibrosis: expert panel discussion on the management of drug-related adverse events. Adv Ther 2014;31:375-391. Erratum in: Adv Ther $2014 ; 31: 575-576$.

40. Oltmanns U, Kahn N, Wenz H, et al. Pirfenidone in idiopathic pulmonary fibrosis-real life experience from a German tertiary referral center for interstitial lung diseases. Respiration. 2014;88:199-207.

41. Cottin V, Maher T. Long-term clinical and real-world experience with pirfenidone in the treatment of idiopathic pulmonary fibrosis. Eur Respir Rev. 2015;24:58-64.

42. Costabel U, Albera C, Bradford WZ, et al. Analysis of lung function and survival in RECAP: an open-label extension study of pirfenidone in patients with idiopathic pulmonary fibrosis. Sarcoidosis Vasc Diffuse Lung Dis. 2014;31:198-205.

43. Hilberg F, Roth GJ, Krssak M, et al. BIBF 1120: triple angiokinase inhibitor with sustained receptor blockade and good antitumor efficacy. Cancer Res. 2008;68:4774-82.

44. Wollin L, Wex E, Pautsch A, et al. Mode of action of nintedanib in the treatment of idiopathic pulmonary fibrosis. Eur Respir J. 2015;45:1434-45. 
45. Keating GM. Nintedanib: a review of its use in patients with idiopathic pulmonary fibrosis. Drugs. 2015;75:1131-40.

46. Rangarajan S, Kurundkar A, Kurundkar D et al. Novel mechanisms for the anti-fibrotic action of nintedanib. Am J Respir Cell Mol Biol 2015 Jun 13. [Epub ahead of print].

47. Richeldi L, Costabel U, Selman M, et al. Efficacy of a tyrosine kinase inhibitor in idiopathic pulmonary fibrosis. N Engl J Med. 2011;365:1079-87.

48. Costabel U, Inoue Y, Richeldi L, et al. Efficacy of nintedanib in idiopathic pulmonary fibrosis across pre-specified subgroups in INPULSIS ${ }^{\circledR}$. Am J Respir Crit Care Med 2015 Sep 22 [epub ahead of print].

49. Corte T, Bonella F, Crestani B, et al. Safety, tolerability and appropriate use of nintedanib in idiopathic pulmonary fibrosis. Respir Res. $2015 ; 16: 116$.

50. Raghu G, Freudenberger TD, Yang S, et al. High prevalence of abnormal acid gastro-oesophageal reflux in idiopathic pulmonary fibrosis. Eur Respir J. 2006;27:136-42.

51. Raghu G, Amatto VC, Behr J, Stowasser S. Comorbidities in idiopathic pulmonary fibrosis patients: a systematic literature review. Eur Respir J. 2015;46:1113-30.

52. Savarino E, Carbone R, Marabotto E, et al. Gastro-oesophageal reflux and gastric aspiration in idiopathic pulmonary fibrosis patients. Eur Respir J. 2013;42:1322-31.

53. Lozo Vukovac E, Lozo M, Mise $\mathrm{K}$, et al. Bronchoalveolar $\mathrm{pH}$ and inflammatory biomarkers in newly diagnosed IPF and GERD patients: a case-control study. Med Sci Monit. 2014;20:255-61.

54. Lee JS, Ryu JH, Elicker BM, et al. Gastroesophageal reflux therapy is associated with longer survival in patients with idiopathic pulmonary fibrosis. Am J Respir Crit Care Med. 2011;184:1390-4.

55. Lee JS, Collard HR, Anstrom KJ, et al. Anti-acid treatment and disease progression in idiopathic pulmonary fibrosis: an analysis of data from three randomised controlled trials. Lancet Resp Med. 2013;1:369-76.

56. Kreuter M, Wuyts W, Renzoni E, et al. Presented at the 25th European Respiratory Society annual meeting, Amsterdam, the Netherlands, September 2015.

57. Spagnolo P, du Bois RM, Cottin V. Rare lung disease and orphan drug development. Lancet Respir Med. 2013;1:479-87.
58. Van Den Blink B, Burggraaf J, Morrison LD, et al. A phase I study of Prm-151 in patients with idiopathic pulmonary fibrosis. Am J Respir Crit Care Med. 2013;187:A5707.

59. Kahloon RA, Xue J, Bhargava A, et al. Patients with idiopathic pulmonary fibrosis with antibodies to heat shock protein 70 have poor prognoses. Am J Respir Crit Care Med. 2013;187:768-75.

60. Sumpter TL, Wilkes DS. Role of autoimmunity in organ allograft rejection: a focus on immunity to type $\mathrm{V}$ collagen in the pathogenesis of lung transplant rejection. Am J Physiol Lung Cell Mol Physiol. 2004;286:L1129-39.

61. Vittal R, Mickler EA, Fisher AJ, et al. Type V collagen induced tolerance suppresses collagen deposition, TGF-beta and associated transcripts in pulmonary fibrosis. PLoS ONE. 2013;8:e76451.

62. Parra ER, Teodoro WR, Velosa AP, et al. Interstitial and vascular type $\mathrm{V}$ collagen morphologic disorganization in usual interstitial pneumonia. J Histochem Cytochem. 2006;54:1315-25.

63. Wilkes DS, Chew T, Flaherty $\mathrm{KR}$, et al. Oral immunotherapy with type $\mathrm{V}$ collagen in idiopathic pulmonary fibrosis. Eur Respir J. 2015;45:1393-402.

64. Nishi Y, Sano H, Kawashima T, et al. Role of galectin-3 in human pulmonary fibrosis. Allergol Int. 2007;56:57-65.

65. Watterson KR, Lanning DA, Diegelmann RF, Spiegel S. Regulation of fibroblast functions by lysophospholipid mediators: potential roles in wound healing. Wound Repair Regen. 2007;15:607-16.

66. Tager AM, LaCamera P, Shea BS, et al. The lysophosphatidic acid receptor LPA1 links pulmonary fibrosis to lung injury by mediating fibroblast recruitment and vascular leak. Nat Med. $2008 ; 14: 45-54$.

67. Raghu G, Scholand MB, De Andrade J, et al. Safety and efficacy of anti-CTGF monoclonal antibody FG-3019 for treatment of idiopathic pulmonary fibrosis (IPF): results of phase 2 clinical trial two years after initiation. Am J Respir Crit Care Med. 2014;189:A1426.

68. Hancock A, Armstrong L, Gama R, Millar A. Production of interleukin 13 by alveolar macrophages from normal and fibrotic lung. Am J Respir Cell Mol Biol. 1998;18:60-5.

69. Belperio JA, Dy M, Burdick MD, et al. Interaction of IL-13 and C10 in the pathogenesis of 
bleomycin-induced pulmonary fibrosis. Am J Respir Cell Mol Biol. 2002;27:419-27.

70. Murray LA, Zhang H, Oak SR, et al. Targeting interleukin-13 with tralokinumab attenuates lung fibrosis and epithelial damage in a humanized SCID idiopathic pulmonary fibrosis model. Am J Respir Cell Mol Biol. 2014;50:985-94.

71. Jakubzick C, Kunkel SL, Puri RK, et al. Therapeutic targeting of IL-4- and IL-13-responsive cells in pulmonary fibrosis. Immunol Res. 2004;30:339-49.

72. Jakubzick C, Choi ES, Carpenter KJ, et al. Human pulmonary fibroblasts exhibit altered interleukin-4 and interleukin-13 receptor subunit expression in idiopathic interstitial pneumonia. Am J Pathol. 2004;164:1989-2001.

73. Soubrane C, Lin Y, Delfolie A, et al. Presented at the 18th international colloquium on lung and airway fibrosis (ICLAF). Quebec: Mont Tremblant; 2014.
74. Barry-Hamilton V, Spangler R, Marshall D, et al. Allosteric inhibition of lysyl oxidase-like-2 impedes the development of a pathologic microenvironment. Nat Med. 2010;16:1009-17.

75. Chien JW, Richards TJ, Gibson KF, et al. Serum lysyl oxidase-like 2 levels and idiopathic pulmonary fibrosis disease progression. Eur Respir J. 2014;43:1430-8.

76. Akhurst RJ, Hata A. Targeting the TGF $\beta$ signalling pathway in disease. Nat Rev Drug Discov. 2012;11:790-811.

77. Horan GS, Wood S, Ona V, et al. Partial inhibition of integrin alpha(v)beta6 prevents pulmonary fibrosis without exacerbating inflammation. Am J Respir Crit Care Med. 2008;177:56-65. 